\title{
On the Posterior Deep Temporal Artery of the Dog
}

\author{
By \\ Fuminiko SUWA, Saisuke TOMINAGA and Hideo KUMAGaI \\ Department of Anatomy, Osaka Dental University; \\ Kyobashi, Higashi-ku, Osaka 540, Japan \\ (Director: Prof. Y. Ohta) \\ -Received for Publication, April 13, 1978-
}

\begin{abstract}
Key Words: Temporal artery, Comparative anatomy.
Summary. The origin and ramification of the posterior deep temporal artery of the dog have been studied by means of the acryl plastic injection method. The artery arose distal to the origin of the inferior alveolar in more than half of all the examples observed, but in common or in contact with the inferior alveolar in others.

All the branches of the artery were mentioned; the lingual, the temporomandibular joint, the anterior, the lateral pterygoid muscular branches and the masseteric artery. Then the artery terminated into the superior, the superoposterior and the posterior branches.
\end{abstract}

Branches of the posterior deep temporal artery of the dog have been briefly reported in works, such as the arterial distribution of the temporal muscle (Fujimoto 1959), the temporomandibular joint (Wakimaru 1959), the medial pterygoid muscle (Tsuji 1969), the masster muscle (Fujiwara 1970) and the mandibular nutrient vessels (Yamamoto et al. 1974). These exclusive works, however, could not include all the ramification and distribution of the artery. yet. Thereupon the present authors have attempted adding a supplement to the previous works by making a further observation about these items and accomplishing the angiology of the artery.

\section{Material and Methods}

Acryl plastic injection was performed on 32 adult dogs, by means of the method of Taniguchi, Ohta and Tajiri (1952 and 1955), through the common carotid arteries. Thirty of the injected heads were treated with sodium hydroxide solution to make corrosion specimens of the carotid system. The other injected heads were preserved in 10\% formalin solution for dissection.

\section{Observations}

The maxillary artery, as a terminus of the external carotid, separating from the superficial temporal artery in the 
mandibular portion, comes right medioinferior to the temporomandibular joint and makes a medial curvature in company with the articulotemporal nerve beneath the postglenoid process. Then the artery runs medially between the posterior margin of the medial pterygoid muscle and the inferior margin of the insertion of the lateral pterygoid toward the alar canal.

From the anterior wall of the curvature, the inferior alveolar artery first is given off anteroinferiorly and the posterior deep temporal artery, 1.2-2.5, M. $1.8 \mathrm{~mm}$ in diameter, superiorly (Fig. $1)$.

The posterior deep temporal in 30 cases of all 60 examples observed arises at $1-4 \mathrm{~mm}$ distal to the origin of the inferior alveolar (Fig. 1) which arises from the anterior wall of the maxillary in all these cases, but the posterior deep temporal arises from the anterior (in 16 cases) or the superior (in 14 cases) wall of the maxillary. In 26 cases of all the examples observed, both arteries arise through a short common stem, 1-2 $\mathrm{mm}$ in diameter, about $2.6 \mathrm{~mm}$ in length, from the anterior wall (Fig. 2). In four cases, both origins of the arteries are located very closely each other (Fig. 3). Any case in which both origins of the arteries are reversed distoproximally, is not observed.

The posterior deep temporal artery which arises in relations with the inferior alveolar artery as mentioned above, immediately hooks upward and enters the insertion of the deep layer of the temporal muscle in the height of the basis of zygomatic process of the temporal bone, after giving off the lingual, the temporomandibular joint, the anterior branches, the lateral pterygoid muscular branch and the masseteric artery (Fujiwara 1970). The artery terminates into the superior, the supero- posterior and the posterior branches (Fujimoto 1959) (Fig. 1). The description of the termini and the masseteric is not made in the present paper as they already did.

1. Lingual branch

The branch, $0.3-1.0$, M. $0.9 \mathrm{~mm}$ in diameter, diverges from the anterior wall of the parent artery, $1-3 \mathrm{~mm}$ distal to the origin of it in all the examples observed. Giving off the pterygoid branches and a branch running along the inferior alveolar nerve (Fig. 1), the main stream passes anteroinferiorly along the superior margin of the lingual nerve between the medial surface of the mandibular ramus and the medial pterygoid muscle on the middle of the venter of it, supplying small twigs to the nerve, and gives off two branches (Fig. 4); one bends medially on the anterior margin of the muscle, then runs posteriorly between the medial surface of it and the mucous membrane and communicates with branches of the facial artery (Fig. 5 ), and the other is close to the anterior margin of the muscle and gives off two or three forward for supplying a mucous plica stringing between the mucous membrane posterior to the upper and lower last molar teeth, also the lingual gingivae of the lower molars and the posterior part of the origin of the mylohyoid muscle. The stream still extends anteroinferiorly along the lingual nerve to supply the submandibular duct and the monostomatic sublingual gland (Miller 1964) (Fig. 4).

(1) R. pterygoideus (Fujimoto 1959, Tsuji 1969)

The ramus, $0.3-0.5$, M. $0.36 \mathrm{~mm}$ in diameter, arises from the superomedial wall of the lingual branch, about $1 \mathrm{~mm}$ distal to its origin, and runs superomedially to supply the insertion of the lateral pterygoid muscle and the posterior margin of the medial pterygoid and the 
medial part of the insertion of the temporal muscle (Figs. 1, 3 and 4).

(2) Branch running along the inferior alveolar nerve.

The branch is $0.2-0.5$, M. $0.32 \mathrm{~mm}$ in diameter. In 45 cases of all 60 examples observed, it arises from the inferior wall of the lingual branch $1-3 \mathrm{~mm}$ distal to its origin in 27 cases (Figs. 1, 4 and 5), from the inferior wall of the inferior alveolar artery right proximal to its origin in 9 cases, from the anterior wall of the posterior deep temporal $1-5 \mathrm{~mm}$ distal to its origin in 8 cases (Fig. 6) and from the anterior wall of the maxillary $1.5 \mathrm{~mm}$ distal to the origin of the inferior alveolar in one case (Fig. 7). Two branches are seen in 15 cases. Each of them arises from the posterior deep temporal and the inferior alveolar, respectively (Fig. 6).

The branch supplies twigs to the inferior alveolar nerve before it enters the mandibular foramen, also the coronoid and the angular branch (Yamamoto et al. 1974) to the contents of the canal including its surrounding bone tissue. It sometimes communicates with the dental branch of $M_{3}$, but does not pass forward beyond the level of the tooth. In 15 cases in which the branch is not seen, a branch arising from the inferior alveolar in the same named canal supplies the nerve as well.

2. Temporomandibular joint branch

The branches, $0.3-0.5$, M. $0.34 \mathrm{~mm}$ in diameter, presenting two in 55 cases and one in 5 cases of all the examples observed, arise posteromedially from the posterior wall of the posterior deep temporal in the height of or distal to the arising of the lingual branch. They supply small twigs to the anterior and medial surfaces of the joint capsule and the insertion of the lateral pterygoid (Figs. 1, 2, 5 and 8).

3. R. anterior (Fujimoto, 1959)
The rami, $0.3-0.5$, M. $0.42 \mathrm{~mm}$ in diameter, presenting two in 57 cases and one in three cases of all the examples observed, arise from the anterior wall of the posterior deep temporal artery anterosuperiorly when the artery hooks upward medioanterior to the capsule. Usually the arisings of them are located between the origins of the lingual branch and the masseteric artery (Figs. 2 and $5)$. The proximal vessel of them arises very close to the origin of the lingual branch or in common with it (Figs. 1 and 3 ).

The rami pass anterosuperiorly far to the inferior end of the insertion of the temporal and supply twigs spreading on the medial surface of the coronoid process.

4. Lateral pterygoid muscular branch

The branch, $0.3-0.6, \quad$ M. $0.38 \mathrm{~mm}$ in diameter, arises from the medial wall of the posterior deep temporal between the origin of the anterior branch and the fork of three termini, but in 31 cases of all the examples observed arises from one of them. The branch, after supplying twigs to the joint and the zygomaticomandibular muscle, runs medially between the insertion of the lateral pterygoid muscle and the basis of the zygomatic process, then anteromedially on the superior margin of the muscle to supply it, anastomosing with the anterior deep temporal.

\section{Discussion}

The posterior deep temporal artery of the dog arose generally from the maxillary just distal to or in common with the inferior alveolar.

Fujimoto (1959), Wakimaru (1959) and the present authors made an attempt to classify relations between the origins of the inferior alveolar and the posterior deep temporal arteries as follows: 1) The 
posterior deep temporal arises distal to the inferior alveolar, 2) through a common trunk, 3). in contact with each other, and 4) the posterior deep temporal arises medial to the inferior alveolar, with excepting that included 3 ) in 1 ) by Wakimaru. The last case 4) was not reported by these scholars. Fujimoto and the present authors observed more examples of 2) and 3) by 4 times than Wakimaru. However, Takarada (1958), Ikakura (1961) and Kubota (1966) reported examples in that the inferior alveolar arose distal to the posterior temporal in approximately $90 \%$ in man, as same as in the dog by Wakimaru, also arose medial to the posterior deep temporal in about 1.5\%. The posterior deep temporal of the pig which arose medial to the inferior alveolar was described in the table by Nickel et al. (1976).

Already the masseteric branch as described in detail by Ellenberger et al. (1891), Bradley (1927), Wakimaru, Miller and Fujiwara (1970), and the termini of the posterior deep temporal which supplied the temporal muscle were done by Fujimoto.

A ramus arising from the inferior alveolar near its origin in man was described as the lingual branch which descended with the lingual nerve and helped to supply the mucous membrane of the mouth in Gray's textbook. The present authors were able to see a ramus that had the same distributing area in the dog as mentioned above, but it arose from the posterior deep temporal artery.

Ellenberger et al. described only that a branch arose from aa. temporales profundae and ran to the arcus palatoglossus along the pterygoid muscle. Fujimoto termed such a ramus the pterygoid muscular branch. The present authors have regarded the ramus as $r$. lingualis of the posterior deep temporal on the basis of the same distributing area.

Yamamoto et al. first made a detailed report on a branch of the posterior deep temporal which was distributed to the mandibular ramus after bifurcating into the angular and coronoid branches. Such a branch in the present paper was described as vasa nervorum of the inferior alveolar nerve, but was not mentioned by them. And the branch in one case did not originate from the posterior deep temporal but from the anterior wall of the maxillary.

The temporomandibular joint branch corresponded to the third joint branch by Wakimaru or the branch supplying the condylar process by Yamamoto et al. The present authors observed that the branch distributed to both the insertion of the lateral pterygoid muscle and the temporomandibular joint, but the branch supplied mainly the muscle, and twigs of the branch anastomosed with a branch of the anterior deep temporal artery, as stated by Fujimoto.

The anterior branch and the termini of the posterior deep temporal were approximately similar to the description of Fujimoto in the distributing area.

The authors wish to express their thanks to Professor Y. Ohta and Assistant Professor T. Tokioka for their excellent advice and encouragement during this study.

\section{Literature cited}

1) Bradley, O.C.: Topographical anatomy of the dog. 2nd ed., Oliver and Boyd, London, 178 \& 179, 1927.

2) Ellenberger, W. und H. Baum: Systematische und topographische Anatomie des Hundes. Paul parey, Berlin, 381-383, 1891.

3) Fujimoto, T.: Cubical anatomy of several ducts and vessels by injection method of acrylic resin. V. Arterial 
distribution of the temporal muscle in some mammals. Okajimas Fol. anat. jap., 33 : 389-424, 1959.

4) Fujiwara, I.: Stereological studies on several ducts and vessels by injection method of acrylic resin. XXVII. Arterial distribution of the masseter muscle in some mammals. Okajimas Fol. anat. jap., 47 : 335-352, 1970.

5) Warwick, R. and P.L. Williams : Gray's Anatomy. 35 th ed, Longman, London, $630,1973$.

6) Ikakura, K.: On the origin, course and distribution of the maxillary artery in Japanese. Kouku kaibou kenkyu, 18 : 91122, 1961. (in Japanese)

7) Kubota, K.: Studies on the nutrient artery of the temporomandibular joint in human embryo. I. The distributing arteries of the temporomandibular joint. Shikagakuho, 66: 765-822, 1966. (in Japanese)

8) Miller, M.E., C.C. Christensen and H.E. Evans: Anatomy of the dog. W. B. Saunder, Phila. and London, 1964.

9) Nickel, R., A. Schummer und E. Seiferle : Lehrbuch der Anatomie der Haustiere. Berlin und Hamburg, Bd. III, 115 \& 116,
1976.

10) Takarada, T.: The anatomical study of the maxillary artery in Japanese. Shikagakuho, $58: 1-20,1958$. (in Japanese)

11) Taniguchi, Y., Y. Ohta and S. Tajiri: New improved method for injection of acrylic resin. Okajimas Fol. anat. jap., 24 : 259-267, 1952.

12) Taniguchi, Y., Ohta, S. Tajiri, H. Okano and H. Hanai: Supplement to new improved method for injection of acrylic resin. Okajimas Fol. anat. jap., 27 : 401406, 1955.

13) Tsuji, T.: Stereological studies on several ducts and vessels by in jection method of acrylic resin. XXII. Arterial distribution of the medial pterygoid muscle in some mammals. Okajimas Fol. anat. jap., 46 : 21-40, 1969.

14) Wakimaru, T.: Studies on the vascular and the nervous systems over the mandibular joint. Md. J. Kagoshima, Univ., 11: 1010-1023, 1959. (in Japanese)

15) Yamamoto, I., T. Yanagawa and A. Arai : On the nutrient arterial branches of the mandibular ramus in the dog by the plastic injection method. Okajimas Fol. anat. jap., 50: 377-392, 1974. 


\title{
Explanation of Figures
}

Key to abbreviations

\author{
F: Mandibular fossa \\ L: Lateral pterygoid muscle \\ $\mathrm{M}$ : Medial pterygoid muscle \\ $\mathrm{T}$ : Temporal muscle \\ i : Inferior alveolar artery \\ $\mathrm{m}$ : Maxillary artery \\ P : Posterior deep temporal artery \\ Branches of the posterior deep temporal artery: \\ an: R. anterior \\ ia: Branch running along the inferior alveolar nerve \\ li : Lingual branch \\ lp: Lateral pterygoid muscular branch \\ ma. Masseteric artery \\ po: Posterior branch \\ pt : R. pterygoideus \\ sp: Superoposterior branch \\ su: Superior branch \\ ti : Temporomandibular joint branch \\ $\leftarrow$ Direction of the snout
}

\section{Plate I}

Fig. 1. Lateral view of the whole aspect of the posterior deep temporal and the maxillary in the mandibular portion of the right side. The insertion of the temporal muscle is indicated by a dotted line. The posterior deep temporal arises from the maxillary distal to the arising of the inferior alveolar, and gives off the lingual branch, the temporomandibular joint branch, the anterior branches and the masseteric (removed) artery. The main stream of the artery spreads into the superior, the posterior and the superoposterior branches to supply the temporal muscle. The lingual branch in this case gives off the pterygoid branches, the other anterior branch $(*)$ and the branch running along the inferior alveolar nerve. ca. $\times 5.0$

Figs. 2 and 3. Lateral view of the arisings of the posterior deep temporal and the inferior alveolar arteries of the left side. In figure 2, both arteries arise through a common trunk. Two joint branches are seen and the anterior branch arises from the posterior deep temporal. In figure 3, both arteries arise in contact with each other. Two anterior branches arise from the posterior deep temporal and the lingual branch, respectively. ca. $\times 4.4, \times 5.0$ 


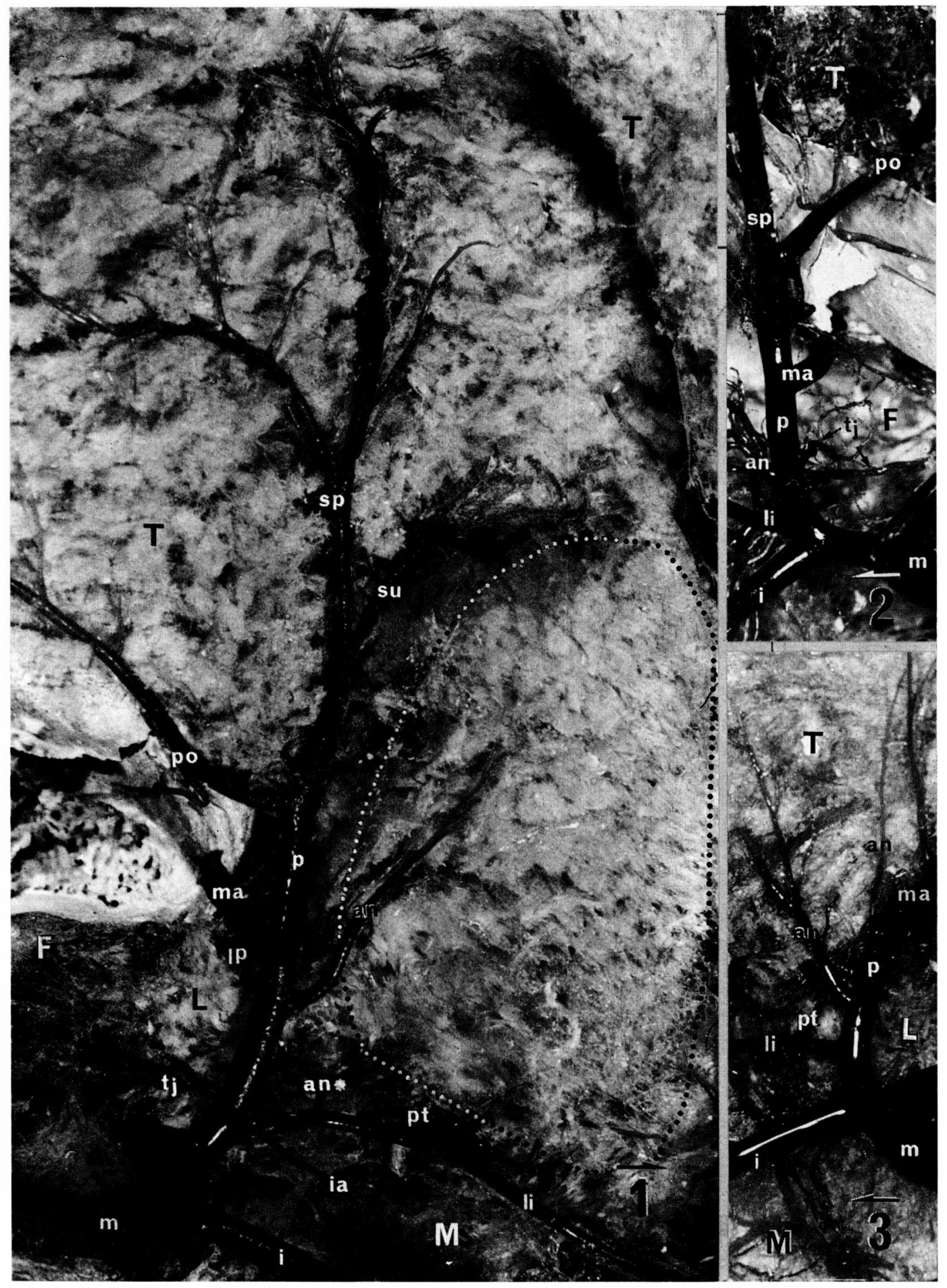




\section{Plate II}

Figs. 4 and 5. Lateral view of the whole aspect of the lingual branch of the left side. Lower teeth and the mandible were removed. The branch arises from the anterior wall of the posterior deep temporal and gives off the anterior and the pterygoid branches upward and the branch running along the inferior alveolar nerve downward. Then it passes anteroinferiorly on the lateral surface of the medial pterygoid muscle where it gives off two twigs; one $(*)$ bending medially at the anterior margin of the muscle to supply it, as in figure 5 extending backward and anastomosing $(\leftarrow)$ with a branch of the facial artery $(\bigcirc)$, and the other (**) supplying a plica of the oral mucous membrane and the lingual gingivae of the lower molar teeth. The main stream extends anteroinferiorly along the lingual nerve to supply the subligual gland $(x)$ and the submandibular duct $(\triangle)$. ca. $\times 3.5, \times 3.6$

Figs. 6, 7 and 8. Lateral view of the arisings of the posterior deep temporal and the inferior alveolar arteries of the right (fig. 6) and the left (figs. 7 and 8) sides. The mandibular foramen is indicated by a dotted line.

In figure 6, The posterior deep temporal and the inferior alveolar arise through a common trunk, and the branch running along the inferior alveolar nerve arises from the former. ca. $\times 3.6$

In figure 7 , the inferior alveolar, the branch running along the inferior alveolar nerve and the posterior deep temporal arise independently from the maxillary in order, and the lingual branch arises from the posterior deep temporal directly. ca. $\times 4.4$

In figure 8 , the posterior deep temporal and the inferior alveolar arise through a common trunk, and two branches running along the inferior alveolar nerve and the lingual branch arise from the inferior alveolar. ca. $\times 4.0$ 
Plate II

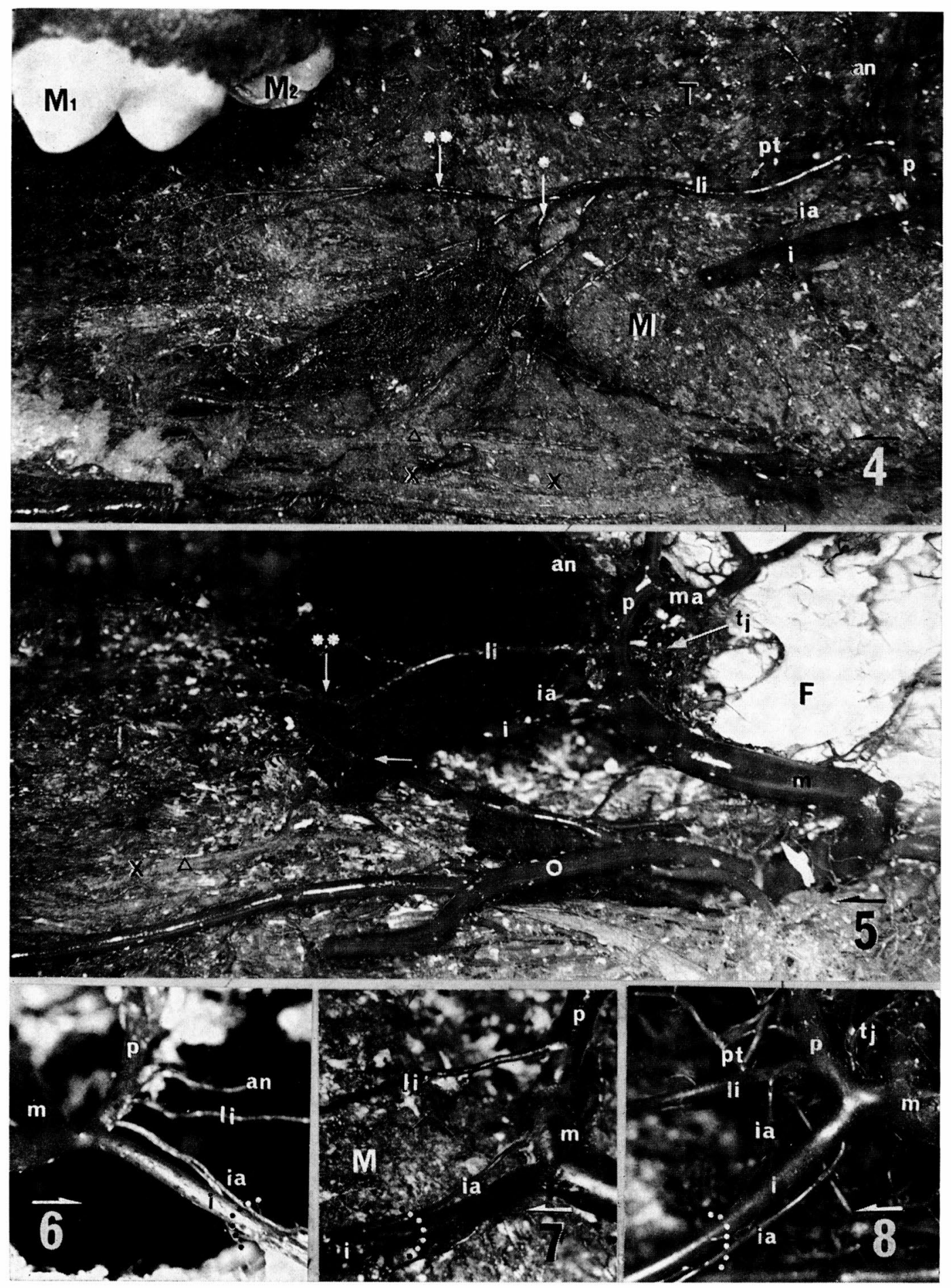

